\title{
On the sets of $n$ points forming $n+1$ directions
}

\author{
Cédric Pilatte \\ Department of Mathematics \\ University of MONS (UMONS) \\ Place du Parc, 20 \\ 7000 Mons, Belgium \\ cedric.pilatte@umons.ac.be
}

Submitted: Nov 11, 2018; Accepted: Dec 22, 2019; Published: Jan 24, 2020

(C) The author. Released under the CC BY-ND license (International 4.0).

\begin{abstract}
Let $S$ be a set of $n \geqslant 7$ points in the plane, no three of which are collinear. Suppose that $S$ determines $n+1$ directions. That is to say, the segments whose endpoints are in $S$ form $n+1$ distinct slopes. We prove that $S$ is, up to an affine transformation, equal to $n$ of the vertices of a regular $(n+1)$-gon. This result was conjectured in 1986 by R. E. Jamison.
\end{abstract}

Mathematics Subject Classifications: 52C10, 52C30, 52C35

\section{Introduction}

In 1970, inspired by a problem of Erdös, Scott [15] asked the following question, now known as the slope problem: what is the minimum number of directions determined by a set of $n$ points in $\mathbb{R}^{2}$, not all on the same line? By the number of directions (or slopes) of a set $S$, we mean the size of the quotient set $\{P Q \mid P, Q \in S, P \neq Q\} / \sim$, where $\sim$ is the equivalence relation given by parallelism: $P_{1} Q_{1} \sim P_{2} Q_{2} \Longleftrightarrow P_{1} Q_{1} \| P_{2} Q_{2}$.

Scott conjectured that $n$ points, not all collinear, determine at least $2\left\lfloor\frac{n}{2}\right\rfloor$ slopes. This bound can be achieved, for even $n$, by a regular $n$-gon; and for odd $n$, by a regular $(n-1)$ gon with its center. After some initial results of Burton and Purdy [2], this conjecture was proven by Ungar [16] in 1982, using techniques of Goodman and Pollack [5]. His beautiful proof is also exposed in the famous Proofs from the Book [1, Chapter 11]. Recently, Pach, Pinchasi and Sharir solved the tree-dimensional analogue of this problem, see[12, 13].

A lot of work has been done to determine the configurations where equality in Ungar's theorem is achieved. A critical set (respectively near-critical set) is a set of $n$ noncollinear points forming $n-1$ slopes (respectively $n$ slopes). Jamison and Hill described four infinite families and 102 sporadic critical configurations $[6,7,10]$. It is conjectured 
that this classification is accurate for $n \geqslant 49$. No classification is known in the near-critical case. See [8] for a survey of these questions, and other related ones.

In this paper, we suppose that no three points of $S$ are collinear (we say that $S$ is in general position). This situation was first investigated by Jamison [9], who proved that $S$ must determine at least $n$ slopes. As above, equality is possible with a regular $n$-gon. It is a well-known fact that affine transformations preserve parallelism. Therefore, the image of a regular $n$-gon under an affine transformation also determines exactly $n$ slopes. ${ }^{1}$ Jamison proved the converse, i.e. that the affinely regular polygons are the only configurations forming exactly $n$ slopes.

A much more general statement is believed to be true: for some constant $c_{1}$, if a set of $n$ points in general position forms $m=2 n-c_{1}$ slopes, then it is affinely equivalent to $n$ of the vertices of a regular $m$-gon (see [9]). This would imply, in particular, that for every $c \geqslant 0$ and $n$ sufficiently large, every simple configuration of $n$ points determining $n+c$ slopes arises from an affinely regular $(n+c)$-gon, after deletion of $c$ points. Jamison's result thus shows it for $c=0$. Here, we will prove the case $c=1$. The general conjecture is still open. In fact, for $c \geqslant 2$, it is not even known whether the points of $S$ form a convex polygon.

Every affinely regular polygon is inscribed in an ellipse. Conics will play an important role in our proof. Another problem of Elekes [3] is the following: for all $m \geqslant 6$ and $C>0$, there exists some $n_{0}(m, C)$ such that every set $S \subset \mathbb{R}^{2}$ with $|S| \geqslant n_{0}(m, C)$ forming at most $C|S|$ slopes contains $m$ points on a (possibly degenerate) conic. It is still unsolved, even for $m=6$.

\section{Preliminary Remarks}

Let $S$ be a set of $n$ points in the plane, in general position, that determines exactly $n+1$ slopes. If $S$ had a point lying strictly inside its convex hull, there would be at least $n+2$ slopes, as was proved by Jamison [9, Theorem 7]. Therefore, we know that we can label the points of $S$ as $A_{1}, \ldots, A_{n}$, such that $A_{1} A_{2} \ldots A_{n}$ is a convex polygon.

For every point $A_{i} \in S$, there are $n-1$ segments, with distinct slopes, joining $A_{i}$ to the other points of $S$. We will say that a slope is forbidden at $A_{i}$ if it is not the slope of any segment $A_{i} A_{j}$, for $j \neq i$. Since $S$ determines $n+1$ slopes, there are exactly two forbidden slopes at each point of $S$.

We will denote by $\nabla A_{i} A_{j}$ the slope of the line $A_{i} A_{j}$. Thus, an equality like $\nabla A_{i_{1}} A_{i_{2}}=$ $\nabla A_{i_{3}} A_{i_{4}}$ is equivalent to $A_{i_{1}} A_{i_{2}} \| A_{i_{3}} A_{i_{4}}$. Throughout our main proof, we will repeatedly make use of the next lemma. It will be particularly useful to prove that a slope is forbidden at a point or that two slopes are equal. As an obvious corollary, we have that $\nabla A_{i-1} A_{i+1}$ is forbidden at $A_{i}$ for all $i \in \mathbb{Z}$. Throughout the paper, when we say "for all $i \in \mathbb{Z}$ ", we consider the indices modulo $n$, so that $A_{n+1}:=A_{1}$, and so on.

\footnotetext{
${ }^{1} \mathrm{~A}$ polygon obtained as the image of a regular polygon by an affine transformation is sometimes called an affine-regular or affinely regular polygon.
} 
Lemma 1. Let $1 \leqslant i<j<k \leqslant n$. Exactly one of the following is true:

- the slope of $A_{i} A_{k}$ is forbidden at $A_{j}$;

- $\exists p, i<p<k$ such that $A_{i} A_{k} \| A_{j} A_{p}$.

Moreover, in the second case, $\nabla A_{j} A_{p} \notin\left\{\nabla A_{i} A_{l} \mid l \neq j, k\right\} \cup\left\{\nabla A_{l} A_{j} \mid l \neq i, k\right\}$.

Proof. This is almost immediate from the definition of a forbidden slope. In the second case, if $p \in\{1, \ldots, n\}$ were not between $i$ and $k$, the segments $A_{i} A_{k}$ and $A_{j} A_{p}$ would intersect. Finally, if $\nabla A_{j} A_{p}$ were equal to some $\nabla A_{i} A_{l}$, then $A_{i} A_{k}\left\|A_{j} A_{p}\right\| A_{i} A_{l}$, so $A_{i}, A_{k}$ and $A_{l}$ would be aligned, a contradiction. The same is true for the segments $A_{l} A_{j}$.

We will also need the following result, which can be found in [14, Chapter 1].

Proposition 2. Let $\mathcal{C}$ be a non-degenerate conic and $O$ a point on $\mathcal{C}$. If $P, Q$ are two points on $\mathcal{C}$, define $P+Q$ to be the unique point $R$ on $\mathcal{C}$ such that $R O \| P Q$ (with the convention that $X X$ is the tangent to $\mathcal{C}$ at $X$, for $X \in \mathcal{C})$. This addition turns $\mathcal{C}$ into an abelian group, of which $O$ is the identity element.

In particular, for $P, Q, R, S$ four points on $\mathcal{C}$, we have $P+Q=R+S$ if and only if $P Q \| R S$. Lemma 3 will enable us to introduce conics in the proof, in order to use proposition 2 .

Lemma 3. Suppose $P_{1}, \ldots, P_{6}$ are points in the plane such that $P_{1} P_{6}\left\|P_{2} P_{5}, P_{2} P_{3}\right\| P_{1} P_{4}$ and $P_{4} P_{5} \| P_{3} P_{6}$. Then $P_{1}, \ldots, P_{6}$ lie on a common conic.

Proof. This follows immediately from Pascal's theorem applied to the hexagon $\mathcal{H}=$ $P_{1} P_{4} P_{5} P_{2} P_{3} P_{6}$. Indeed, the intersections of the opposite sides of $\mathcal{H}$ are collinear on the line at infinity.

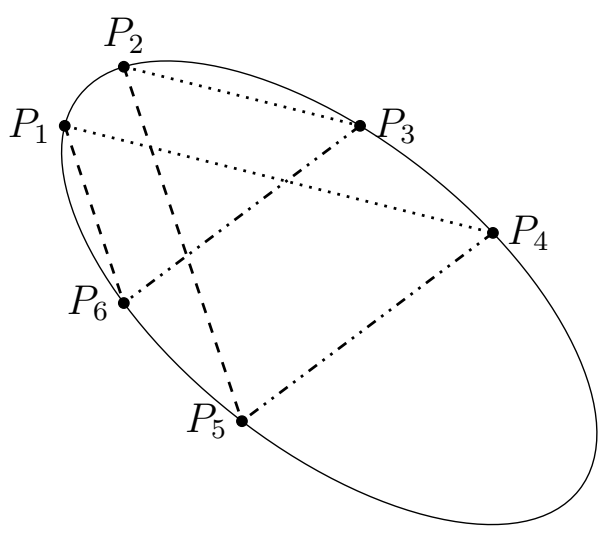

Figure 1: Illustration of lemma 3.

For the reader's convenience, we reproduce here a result of Korchmáros [11] (which is also discussed in [4]), that we will use twice in the proof.

Lemma 4. Let $P_{1}, \ldots, P_{n}$ be distinct points on a non-degenerate conic. Suppose that, for all $j \in \mathbb{Z}, P_{j+1} P_{j+2} \| P_{j} P_{j+3}$. Then, $P$ is affinely equivalent to a regular $n$-gon. 


\section{Main Theorem}

In this section, we prove the following theorem, using the results from section 2.

Theorem 5. Any set $S$ of $n \geqslant 7$ points in the plane, in general position, that determines exactly $n+1$ slopes, is affinely equivalent to $n$ of the vertices of a regular $(n+1)$-gon.

Proof. We use the notations of section 2: $S=\left\{A_{1}, A_{2}, \ldots, A_{n}\right\}$ where $A_{1} A_{2} \ldots A_{n}$ is a convex polygon. We will split the proof into two cases. In the first case, we suppose that, for every $i \in \mathbb{Z}, A_{i+1} A_{i+2} \| A_{i} A_{i+3}$. If this fails for some $i$, we can assume that this $i$ is 1 .

Case 1 For every $i \in \mathbb{Z}, A_{i+1} A_{i+2} \| A_{i} A_{i+3}$.

We will distinguish subcases according to which segments are parallel to $A_{i} A_{i+5}$. As we will see, none of the subcases are actually possible.

$\underline{\text { Case } 1.1}$ For all $i \in \mathbb{Z}, A_{i} A_{i+5} \| A_{i+1} A_{i+4}$.

Let $A_{k+1}, \ldots, A_{k+6}$ be any six consecutive points of $S$. We have $A_{k+1} A_{k+6} \| A_{k+2} A_{k+5}$, $A_{k+2} A_{k+3} \| A_{k+1} A_{k+4}$ and $A_{k+4} A_{k+5} \| A_{k+3} A_{k+6}$ from our two assumptions. Thus, lemma 3 implies that the six points lie on a common conic. As this is true for any six consecutive points, and since five points in general position determine a unique conic, all the $A_{i}$ 's lie on the same conic. Together with the fact that $\forall i, A_{i+1} A_{i+2} \| A_{i} A_{i+3}$, this implies that $A_{1} A_{2} \ldots A_{n}$ is affinely equivalent to a regular $n$-gon, by lemma 4 . Therefore, $S$ determines exactly $n$ directions, which is a contradiction.

Case 1.2 For some $i \in \mathbb{Z}$, we have $A_{i} A_{i+5} \| A_{i+2} A_{i+4}$.

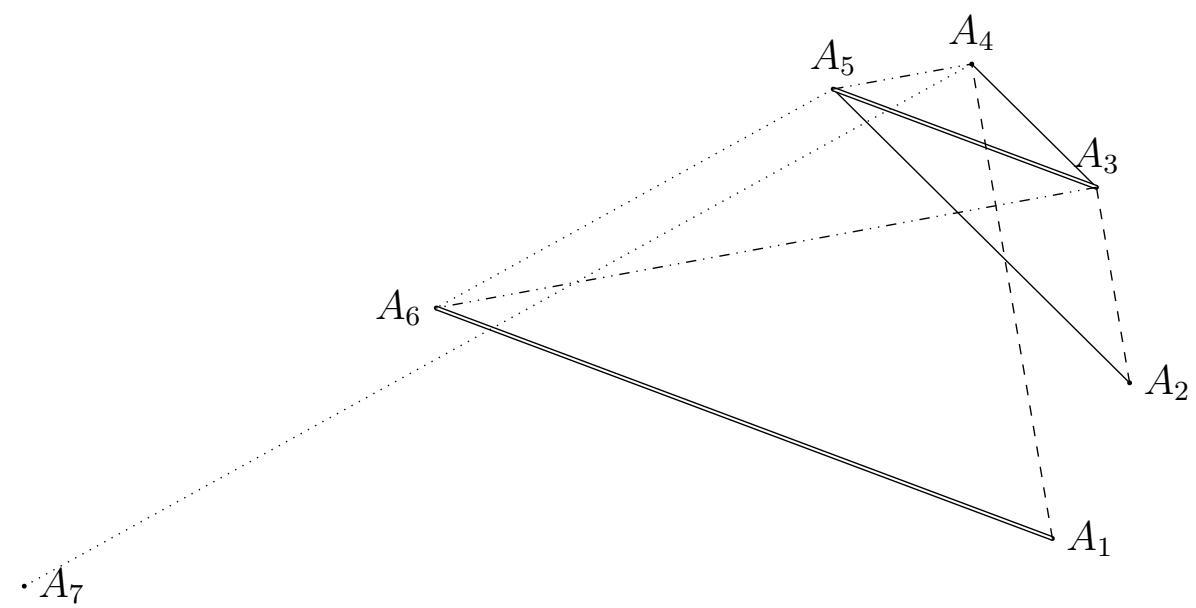

Figure 2: Case 1.2.

Say $i=1$, meaning $A_{1} A_{6} \| A_{3} A_{5}$. By lemma 1 applied three times, we see that $\nabla A_{2} A_{6}$ is forbidden at $A_{3}, A_{4}$ and $A_{5}$ (here, we have used that $A_{2} A_{6} \nVdash A_{3} A_{5}$ and, for $A_{4}$, that $A_{3} A_{4} \| A_{2} A_{5}$ and $\left.A_{4} A_{5} \| A_{3} A_{6}\right)$. For $l=3,4,5$, we know that $\nabla A_{2} A_{6}$ and $\nabla A_{l-1} A_{l+1}$ are exactly the two forbidden slopes at $A_{l}$. Therefore, $\nabla A_{1} A_{5}$ is not forbidden 
at $A_{4}$, hence, by lemma 1 again, we conclude that $A_{1} A_{5} \| A_{2} A_{4}$. Similarly, $\nabla A_{3} A_{7}$ is not forbidden at $A_{4}$, so $A_{3} A_{7} \| A_{4} A_{6}$. As the slope of $A_{2} A_{7}$ is not forbidden at $A_{5}$, we conclude $A_{2} A_{7} \| A_{4} A_{5}\left(\| A_{3} A_{6}\right)$. We have $A_{3} A_{4}\left\|A_{2} A_{5}, A_{3} A_{6}\right\| A_{2} A_{7}$ and we just showed that $A_{2} A_{7} \| A_{3} A_{6}$. By lemma $3, A_{2}, A_{3}, \ldots, A_{7}$ lie on a common conic.

We will equip this conic with the group structure descibed in lemma 2 , with $A_{7}$ the zero element. We will write $A_{7}=0$ and $A_{6}=x$. Then, $A_{5} A_{6}\left\|A_{4} A_{7}, A_{4} A_{5}\right\| A_{3} A_{6}$ and $A_{4} A_{6} \| A_{3} A_{7}$ together imply $A_{5}=2 x, A_{4}=3 x$ and $A_{3}=4 x$. Also, $A_{3} A_{4} \| A_{2} A_{5}$ gives $A_{2}=5 x$. Let $B$ be the point on the conic with $B=6 x$. We thus have $A_{2} A_{3} \| B A_{4}$ and $A_{2} A_{4} \| B A_{5}$. However, there can only be one point $P$ with $A_{2} A_{3} \| P A_{4}$ and $A_{2} A_{4} \| P A_{5}$. As $A_{1}$ is such a point, $A_{1}=B=6 x$. This contradicts $A_{1} A_{6} \| A_{3} A_{5}$, as $A_{1}+A_{6}=6 x+x \neq 4 x+2 x=A_{3}+A_{5}$.

Case 1.3 For some $i \in \mathbb{Z}$, we have $A_{i} A_{i+5} \| A_{i+1} A_{i+3}$.

This is exactly the previous case after having relabelled every $A_{i}$ as $A_{n+1-i}$.

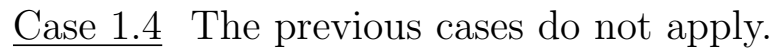

If none of the previous cases is possible, there must be some $i$, say $i=1$, for which $A_{1} A_{6}$ is not parallel to any of $A_{2} A_{5}, A_{3} A_{5}$ and $A_{2} A_{4}$. Then, $\nabla A_{1} A_{6}$ is forbidden at $A_{2}, A_{3}, A_{4}$ and $A_{5}$. Once again, we deduce that the forbidden slopes at $A_{l}, 2 \leqslant l \leqslant 5$, are $\nabla A_{1} A_{6}$ and $\nabla A_{l-1} A_{l+1}$. We use lemma 1 to find $A_{2} A_{6} \| A_{3} A_{5}$ (applied with $A_{k}=A_{4}$ ) and $A_{1} A_{5} \| A_{2} A_{4}\left(A_{k}=A_{2}\right)$.

Let $\mathcal{C}$ be the conic passing through $A_{1}, A_{2}, \ldots, A_{5}$. We use lemma 2 to define a group structure on $\mathcal{C}$, with $A_{1}=0$. Let $A_{2}=x$ and $A_{3}=y$. From $A_{2} A_{3} \| A_{1} A_{4}$ and $A_{3} A_{4} \| A_{2} A_{5}$, we have $A_{4}=x+y$ and $A_{5}=2 y$. But $A_{2} A_{4} \| A_{1} A_{5}$ implies $y=2 x$, so $A_{i}=(i-1) x$ for $1 \leqslant i \leqslant 5$. We use the same argument as before. Let $B=5 x$, then $A_{4} A_{5} \| A_{3} B$ and $A_{3} A_{5} \| A_{2} B$, so $B=A_{6}=5 x$. We deduce $A_{1} A_{6} \| A_{2} A_{5}$, a contradiction.

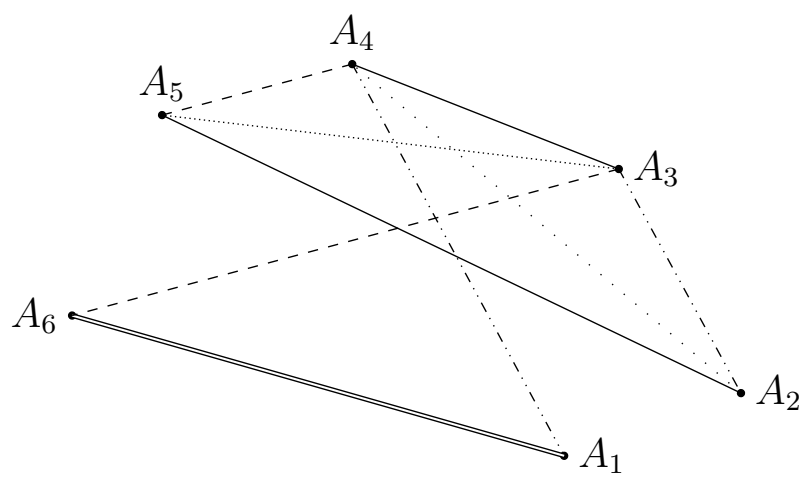

Figure 3: Case 1.4. 
Case 2 We have $A_{2} A_{3} \nVdash A_{1} A_{4}$ (without loss of generality).

Without loss of generality, we can also suppose that the point $A_{4}$ is closer to the line $A_{2} A_{3}$ than is $A_{1}$. In this situation, the line parallel to $A_{2} A_{3}$ passing through $A_{4}$ intersects the segment $\left[A_{1} A_{2}\right]$ in its relative interior, and the line parallel to $A_{2} A_{3}$ passing through $A_{1}$ does not intersect the segment $\left[A_{3} A_{4}\right]$.

From $A_{2} A_{3} \nVdash A_{1} A_{4}$, we deduce that the forbidden slopes at $A_{2}$ and $A_{3}$ are $\nabla A_{1} A_{3}$, $\nabla A_{1} A_{4}$ and $\nabla A_{2} A_{4}, \nabla A_{1} A_{4}$, respectively. Thus, $A_{1} A_{2} \| A_{n} A_{3}$ and $A_{2} A_{5} \| A_{3} A_{4}$. We now show that $A_{2} A_{3}$ is forbidden at $A_{4}$. Suppose, for some $k$, that $A_{2} A_{3} \| A_{4} A_{k}$. Then, $k$ has to be between 5 and $n$, so $A_{1} A_{2} A_{3} A_{4} A_{k}$ must be a convex polygon, with $A_{2} A_{3} \| A_{4} A_{k}$. We can see that this contradicts the fact that $A_{4}$ is closer than $A_{1}$ to the line $A_{2} A_{3}$.

Case $2.1 A_{n-1} A_{2} \| A_{n} A_{1}$.

We want to show that this case is impossible. From lemma 1, we find $A_{n-1} A_{3} \| A_{n} A_{2}$. When we apply this lemma again with the slope of $A_{n} A_{4}$, we find that $A_{n} A_{4}$ is parallel to $A_{1} A_{3}$, because $A_{2} A_{3}$ is forbidden at $A_{4}$. In the same way, we get $A_{n-1} A_{4} \| A_{n} A_{3}$.

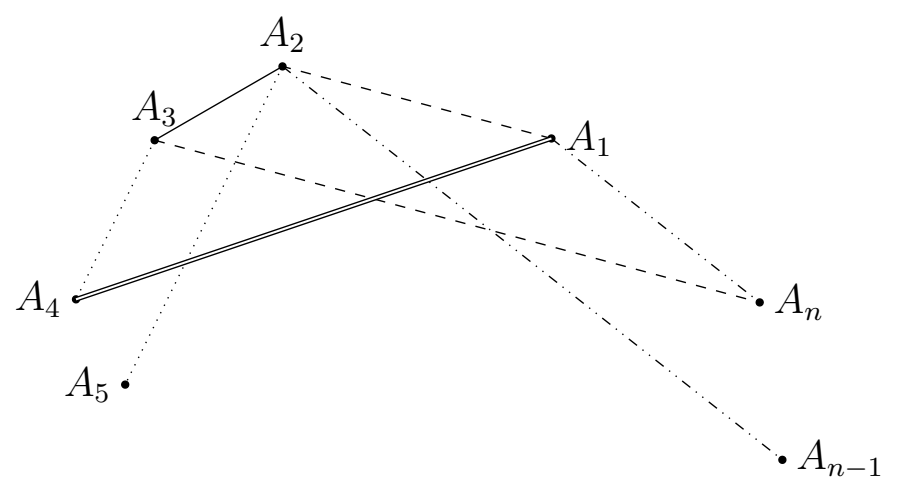

Figure 4: Case 2.1.

Let $\mathcal{C}$ be the conic passing through $A_{3}, A_{2}, A_{1}, A_{n}$ and $A_{n-1}$. Again, we use proposition 2, setting $A_{n-1}=0$. Let $A_{n}=x$ and $A_{2}=y$. From $A_{n-1} A_{3} \| A_{n} A_{2}$ we deduce $A_{3}=x+y$, and from $A_{n-1} A_{4} \| A_{n} A_{3}$ we get $A_{4}=y+2 x$. Let $B=2 x$. Then $A_{n} A_{4} \| B A_{3}$ and $A_{n} A_{3} \| B A_{2}$. This means that $B$ belongs to the line parallel to $A_{n} A_{4}$ through $A_{3}$ and to the line parallel to $A_{n} A_{3}$ through $A_{2}$. So $B=A_{1}$, i.e. $A_{1}=2 x$. On the one hand, the relation $A_{n-1} A_{2} \| A_{n} A_{1}$ gives $0+y=x+2 x$. On the other hand, $A_{2} A_{3} \nVdash A_{1} A_{4}$ yields $y+(y+x) \neq 2 x+(y+2 x)$. This is a contradiction.

Case $2.2 A_{n-1} A_{2} \nVdash A_{n} A_{1}$.

This is the last case of the proof, and the only case that produces valid configurations of points. As $A_{n-1} A_{2} \nVdash A_{n} A_{1}, \nabla A_{n-1} A_{2}$ is forbidden at $A_{1}$. With $\nabla A_{0} A_{2}$, those are the two forbidden slopes at $A_{1}$. Therefore, none of $\nabla A_{2} A_{i}, 3 \leqslant i \leqslant n-2$ is forbidden at $A_{1}$. So, every $\nabla A_{2} A_{i}, 3 \leqslant i \leqslant n-2$, corresponds to a unique $\nabla A_{1} A_{j}$ for some $j$. 


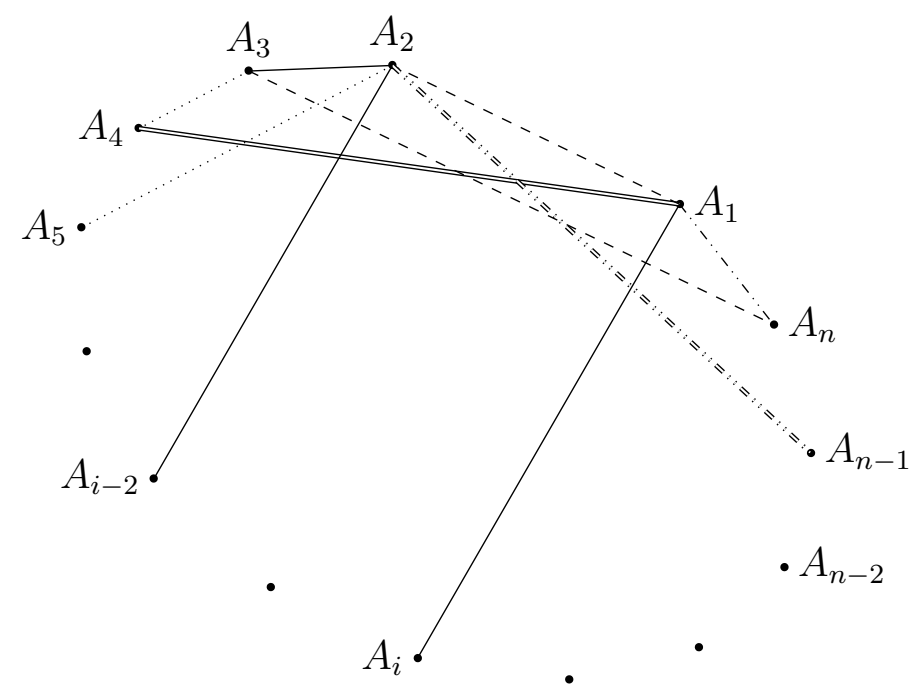

Figure 5: Case 2.2.

A simple but important observation is that, for all $3 \leqslant i_{1}, i_{2} \leqslant n-2$ and $4 \leqslant j_{1}, j_{2} \leqslant n$,

$$
\left\{\begin{array}{l}
A_{2} A_{i_{1}} \| A_{1} A_{j_{1}} \\
A_{2} A_{i_{2}} \| A_{1} A_{j_{2}}
\end{array} \quad \Longrightarrow \quad\left(i_{1}<i_{2} \Longleftrightarrow j_{1}<j_{2}\right) .\right.
$$

That is, the assignment $f$ that maps every $3 \leqslant i \leqslant n-2$ to the unique $4 \leqslant j \leqslant n$ such that $A_{2} A_{i} \| A_{1} A_{j}$ must be strictly increasing. Moreover, it has to satisfy $f(3) \neq 4$ as we assumed $A_{2} A_{3} \nVdash A_{1} A_{4}$. The unique possibility is then $f(i)=i+2$ for every $i$. We have proven that, for $5 \leqslant i \leqslant n, A_{2} A_{i-2} \| A_{1} A_{i}$.

Claim 2.2.1. For every $i \in\{5, \ldots, n\}$,

1. $A_{i-2} A_{i}$ and $A_{2} A_{i-2}$ are the two forbidden slopes at $A_{i-1}$, and;

2. $\forall k \in\{3, \ldots, i-2\}, A_{i-1} A_{k} \| A_{i} A_{k-1}$.

Proof of claim. For $i=5$, we have already proven those two statements. We will prove them for $i=j$, assuming it has already been proven for all $5 \leqslant i \leqslant j-1$.

1. We have to show that $A_{2} A_{j-2}$ is forbidden at $A_{j-1}$. This is clear as $A_{2} A_{j-2} \| A_{1} A_{j}$ and there is no point of $S$ between $A_{1}$ and $A_{2}$.

2. Since we know the forbidden slopes at $A_{j-1}$, we can use lemma 1 at the point $A_{j-1}$ several times, with different slopes. First, $\nabla A_{j} A_{j-3}$ is not forbidden, so $A_{j} A_{j-3}$ and $A_{j-1} A_{j-2}$ are parallel. Then $\nabla A_{j} A_{j-4}$ is not forbidden, and is distinct from $\nabla A_{j-1} A_{j-2}=\nabla A_{j} A_{j-3}$, so $A_{j} A_{j-4} \| A_{j-1} A_{j-3}$. We can continue this way, until we get $A_{j} A_{2} \| A_{j-1} A_{3}$. This concludes the proof of the claim.

In particular, for every $i \in\{6, \ldots, n-1\}$, we have $A_{3} A_{i}\left\|A_{2} A_{i+1}, A_{5} A_{i}\right\| A_{4} A_{i+1}$. As $A_{3} A_{4} \| A_{2} A_{5}$, we can use lemma 1 , which shows that $A_{2}, A_{3}, A_{4}, A_{5}, A_{i}$ and $A_{i+1}$ lie on a conic. As this is true for every $6 \leqslant i \leqslant n-1$, we know that the $A_{i}$ 's, for $2 \leqslant i \leqslant n$, 
all lie on a common conic (because there is a unique conic passing through five points in general position).

As we have done several times in this proof, we use the group structure on the conic given by parallelism. Choose $A_{2}$ to be the identity element, let $A_{3}=x$. Solving

$$
\left\{\begin{array}{l}
A_{3} A_{4} \| A_{2} A_{5} \\
A_{3} A_{6} \| A_{4} A_{5} \\
A_{3} A_{5} \| A_{2} A_{6}
\end{array}\right.
$$

gives $A_{4}=2 x, A_{5}=3 x$ and $A_{6}=4 x$. Then, a simple induction (using $A_{i-1} A_{3} \| A_{i} A_{2}$ ) gives $A_{i}=(i-2) x$ for all $i \in\{2, \ldots, n\}$. Let $B$ be the point on the conic with $B=-2 x$. Then $A_{2} A_{3} \| B A_{5}$ and $A_{2} A_{4} \| B A_{6}$. However, we proved before that $A_{2} A_{3} \| A_{1} A_{5}$ and $A_{2} A_{4} \| A_{1} A_{6}$, so $A_{1}=B=-2 x$.

To summarize, we know that all the $n$ points of $S$ are on a conic, $A_{i}=(i-2) x$ for $i \in\{2, \ldots, n\}$ and $A_{1}=-2 x$. We use the group structure one last time: $A_{3} A_{n} \| A_{1} A_{2}$ implies $x+(n-2) x=-2 x+0$, so $(n+1) x=0$. Therefore, the subgroup generated by $A_{3}=x$ is a finite cyclic group of order $n+1$ :

$$
\left\langle A_{3}\right\rangle=\{\underbrace{A_{2}}_{0}, \underbrace{A_{3}}_{x}, \underbrace{A_{4}}_{2 x}, \ldots, \underbrace{A_{n-1}}_{(n-3) x}, \underbrace{A_{n}}_{(n-2) x}, \underbrace{A_{1}}_{(n-1) x},-x\} .
$$

To finish the proof, we use the more convenient notations $P_{j}:=j x$ for $0 \leqslant j \leqslant n$ (so that every $A_{i}$ is a $P_{j}$ ). If the indices are considered modulo $n+1$, we have, for all $j \in \mathbb{Z}$, $P_{j+1} P_{j+2} \| P_{j} P_{j+3}$, because $(j+1) x+(j+2) x=j x+(j+3) x$. By lemma $4, P_{0} P_{1} P_{2} \ldots P_{n}$ is, up to an affine transformation, a regular $(n+1)$-gon.

\section{Acknowledgements}

I would like to thank Christian Michaux, without whom this work would not have been possible.

\section{References}

[1] Martin Aigner and Günter Ziegler. Proofs from the Book, volume 274. Springer, 2010.

[2] G. R. Burton and G. B. Purdy. The directions determined by $n$ points in the plane. Journal of the London Mathematical Society, 2(1):109-114, 1979.

[3] György Elekes. On linear combinatorics III. Few directions and distorted lattices. Combinatorica, 19(1):43-53, 1999.

[4] J. Chris Fisher and Robert E. Jamison. Properties of affinely regular polygons. Geometriae Dedicata, 69(3):241-259, 1998.

[5] Jacob E. Goodman and Richard Pollack. A combinatorial perspective on some problems in geometry. Congressus Numerantium, 32(0981):383-394, 1981. 
[6] Robert E. Jamison. Planar configurations which determine few slopes. Geometriae Dedicata, 16(1):17-34, 1984.

[7] Robert E. Jamison. Structure of slope-critical configurations. Geometriae Dedicata, 16(3):249-277, 1984.

[8] Robert E. Jamison. A survey of the slope problem. Annals of the New York Academy of Sciences, 440(1):34-51, 1985.

[9] Robert E. Jamison. Few slopes without collinearity. Discrete Mathematics, 60:199206, 1986.

[10] Robert E. Jamison and Dale Hill. A catalogue of sporadic slope-critical configurations. Congressus Numerantium, 40:101-125, 1983.

[11] G. Korchmáros. Poligoni affin-regolari dei piani di galois d'ordine dispari. Atti Accad. Naz. Lincei Rend. Cl. Sci. Fis. Mat. Natur.(8), 56(5):690-697, 1974.

[12] János Pach, Rom Pinchasi, and Micha Sharir. On the number of directions determined by a three-dimensional points set. Journal of Combinatorial Theory, Series A, 108(1):1-16, 2004.

[13] János Pach, Rom Pinchasi, and Micha Sharir. Solution of scott's problem on the number of directions determined by a point set in 3-space. Discrete $\mathscr{E}$ Computational Geometry, 38(2):399-441, 2007.

[14] Viktor V. Prasolov and Yuri P. Solovyev. Elliptic functions and elliptic integrals, volume 170. American Mathematical Soc., 1997.

[15] Paul R. Scott. On the sets of directions determined by $n$ points. The American Mathematical Monthly, 77(5):502-505, 1970.

[16] Peter Ungar. $2 n$ noncollinear points determine at least $2 n$ directions. Journal of Combinatorial Theory, Series A, 33(3):343-347, 1982. 